\title{
Effects of Tempol in Lipopolysaccharide-Induced Liver Injury
}

\author{
Perihan Sinem Serin $^{1}$ (D), Asli Kandil ${ }^{2}$ (D) Huri Bulut ${ }^{3}{ }^{(D)}$ Tugba Kaskavalci $^{4}$ (D), \\ Erman Caner Bulut ${ }^{4}$ (D), Cihan Demirci-Tansel ${ }^{2}$ (D)
}

\author{
'Yeditepe University Specialty Hospital, Adult Bone Marrow Transplantation Unit, Istanbul, Turkey \\ ${ }^{2}$ Istanbul University, Faculty of Science, Department of Biology, Istanbul, Turkey \\ ${ }^{3}$ Bezmialem Vakif University, Faculty of Medicine, Department of Medical Biochemistry, Istanbul, Turkey \\ ${ }^{4}$ Istanbul University, Institute of Graduate Studies in Sciences, Department of Biology, Istanbul, Turkey
}

ORCID IDs of the authors: P.S.S. 0000-0001-6404-5687; A.K. 0000-0001-8408-2610; H.B. 0000-0003-2706-9625; T.K. 0000-0002-4938-9702; E.C.B. 0000-0001-8674-0947; C.D.T. 0000-0001-7926-3089

Please cite this article as: Serin PS, Kandil A, Bulut H, Kaskavalci T, Bulut EC, Demirci-Tansel C. Effects of Tempol in Lipopolysaccharide-Induced Liver Injury. Eur J Biol 2019; 78(2): 147-155. DOI: 10.26650/EurJBiol.2019.0034

\begin{abstract}
Objective: Sepsis leads to conditions such as inflammatory and anti-inflammatory process, circulatory abnormalities, cellular and humoral reactions. Endotoxin-induced oxidative stress causes injury in the liver. The aim of this study was to evaluate the effects of a radical scavenger Tempol in lipopolysaccharide (LPS)-induced liver injury in rats.

Materials and Methods: Male Wistar rats were divided into four groups: Control, LPS (15 mg/kg), LPS + Tempol group (100 $\mathrm{mg} / \mathrm{kg}$ Tempol, three hours after LPS administration) and Tempol (100 mg/kg). Blood glucose and body temperature were measured during the experiment. Superoxide dismutase (SOD), aspartate aminotransferase (AST), alanine aminotransferase (ALT) and C-reactive protein (CRP) levels were measured in plasma or liver tissue. Furthermore, histopathological changes and myeloperoxidase-stained leukocytes infiltration were assessed in liver tissue.

Results: LPS caused tissue damage and leukocytes infiltration, increased AST, ALT and CRP levels, and decreased body temperature, blood glucose and SOD levels. Tempol reduced AST and ALT levels and increased SOD levels. Tempol did not prevent tissue damage, leukocytes infiltration and increment of CRP levels. There were no changes in body temperature and blood glucose levels.

Conclusion: The present study suggests that tempol may have antioxidant properties in LPS-induced liver injury. These results may contribute to a better understanding of the role of tempol and basic mechanisms of underlying oxidative stressrelated liver injury for further investigations.
\end{abstract}

Keywords: Lipopolysaccharide, Liver, Tempol

\section{INTRODUCTION}

Sepsis is characterized by hypotension, vascular hyporeactivity to vasoconstrictor agents, distribution of organ blood flow and myocardial dysfunction $(1,2)$. Sepsis is caused by gram negative and gram positive bacteria ( 2 , 3). It is known to cause the release of cytokines, activation of pro- and anti-inflammatory pathways, coagulation and endothelial activation, and then lead to multiple organ failure (1-4). The liver has an important role in the initiation and progression of multiple organ failure in sepsis and shock (5-8). Pathophysiology of liver injury is complex, and not yet fully understood. Liver dysfunction occurs depending on hepatic blood flow perfusion deterioration and hypoxia in relation to systemic and microvascular circulation failures $(6,9,10)$, direct damage of endotoxins such as lipopolysaccharide (LPS) (6), inflammation and cytokines (9-14).

The liver which contains hepatocytes, Kupffer cells and sinusoidal endothelial cells regulates both metabolic and immunological signal pathways, and has a critical role in the inflammatory responses in infection $(6,15)$. Kupffer cells are the primary defenders of the liver, and prevent 
the entry of bacteria and endotoxins into the portal vein from systemic circulation $(9,10,15-17)$. Kupffer cells are activated by LPS which causes the production of cytokines, nitric oxide and reactive oxygen species (ROS) $(10,13,18)$. Kupffer cells stimulate neighbouring cells including endothelial cells, polymorphonuclear leukocytes, thrombocytes, lymphocytes, Ito cells and hepatic parenchymal cells to secrete various pro-inflammatory cytokines and chemical agents which induce endothelial cell and hepatocyte damage $(2,6,10,13,18)$. In addition to hepatocytes damage, leukocytes, thrombocytes, Kupffer and other inflammatory cells because activated endothelial cells causes damage in sinusoids and leads to fibrin microtrombin formation (14). The increase and aggregation of these inflammatory cells into sinusoids cause occlusion and lead to the decrease of blood flow in sinusoids $(14,19)$. This gives rise to structural changes in the sinusoidal endothelial cells, hepatocytes and bile canaliculi membranes, and expansion of the Disse areas.

The increased level of endotoxin in circulation has a key role in the release of cytokines and systemic inflammatory response. The formation of free radicals and ROS can also be a trigger in the case of the inflammatory response resulting from the endotoxin such as LPS (3). These free radicals are neutralized by complex antioxidant systems in the normal physiological station $(3,20)$. Antioxidant enzymes are superoxide dismutase (SOD), glutathione peroxidase catalase, hydroperoxidase and cytochrome $C$ oxidase. SOD catalyzes superoxide free radical to water and oxygen, and mainly detoxifies ROS in cells $(3,20,21)$.

Tempol (4-hydroxy-2,2,6,6-tetramethylpiperidine-1-oxy) is a scavenger eliminating the formations and effects of many free radicals such as superoxide anions, peroxynitrite and hydroxyl radicals. Tempol is a low molecular weight, stable nitroxide and cell membrane-permeable SOD mimetic compound (22). Moreover, tempol is widely studied in the experimental animal models (23-27). Many previous studies have shown the beneficial effects of tempol in endotoxemia and sepsis (27-31). It has been suggested that tempol protected renal blood flow and glomerular filtration rate $(28,29)$, inhibited damages in kidney and liver, maintained blood flow and metabolism $(30,31)$ and improved mesenteric blood flow (32) in experimental shock models.

The aim of this study was to evaluate the effects of tempol on oxidative stress, tissue damage and inflammation in LPS-induced liver injury.

\section{MATERIALS and METHODS}

\section{Animals}

The present study was approved by Istanbul University Local Committee on animal Research Ethics. Care and handling of animals were in accordance with the Institute for Laboratory Animal Research Guide for Care and Use of Laboratory Animals. Experiments were performed on 24 Wistar albino male rats (Bezmialem Vakif University, Istanbul, Turkey) with body weight of 300-350g.

\section{Experimental Protocol}

The animals were divided into 4 groups $(n=6)$.

Control group: Isotonic saline solution was administered intraperitoneally (ip).

LPS group: 15 mg/kg LPS (E. coli, serotype 026: B6, Sigma, Missouri, USA) was administered ip.

LPS + Tempol group: 100 mg/kg Tempol (4-hidroksi-TEMPO), Sigma, Missouri, USA) was injected ip 3 hours after LPS injection.

Tempol group: $100 \mathrm{mg} / \mathrm{kg}$ Tempol was administrated ip 3 hours after isotonic saline solution injection.

The animals were anesthetized with intraperitoneal injection of sodium pentothal ( $90 \mathrm{mg} / \mathrm{kg}$, I.E ULAGAY, Istanbul, Turkey). Blood samples were collected by cardiac puncture. Liver samples were taken for histological, immunohistochemical and biochemical analyses.

\section{Body Temperature and Blood Glucose Measurement}

Blood samples were taken from the tail vein to measure blood glucose level at three time points 1 ) the beginning of experiment (baseline, T0); 2) third hour of experiment (the middle of experiment, T1); 3) sixth hour of experiment (the end of experiment, T2). In addition, the body temperature of animals in each group was measured by rectal Probe for rats (ADInstruments, Sidney, AUS) at T0, T1 and T2 time points.

\section{Biochemical Analyses}

Blood samples were centrifuged to collect plasma at 4000xg for 10 minutes, at $4^{\circ} \mathrm{C}$. The plasma samples were stored at $-20^{\circ} \mathrm{C}$ until biochemical analyses. Liver samples were weighed (wet weight) and homogenized in Sorensen's phosphate buffer (pH 7.4) with homogenizer (Sartorius, Goettingen, Germany). Homogenates were diluted $1 / 10(\mathrm{w} / \mathrm{v})$ and then centrifuged at $4000 \mathrm{rpm}$ for 7 minutes at $4^{\circ} \mathrm{C}$. Supernatants were stored at $-20^{\circ} \mathrm{C}$ until analysis.

SOD, aspartate aminotransferase (AST), alanine aminotransferase (ALT) and C-reactive protein (CRP) levels were measured in plasma and/or liver tissue using ELISA kits for rats (Uscn Life Science Inc., Qingdao, Shandong, China) according to the manufacturer's protocol.

\section{Histological Analysis}

Liver samples were fixed in $10 \%$ neutral formalin and embedded in paraffin. Liver sections $(4 \mu \mathrm{m})$ were taken with a Rotary microtome and stained with hematoxylin-eosin (HE). Histopathological changes in liver tissue were examined under a light microscope. Photographs of the sections were taken with Image Pro-Plus (Kameram 390CU, Istanbul, Turkey).

\section{Immunohistochemical Analysis}

Liver samples were fixed in 10\% neutral formalin, embedded in paraffin and prepared as described previously (33). Myeloperoxidase (MPO)-staining was performed on the liver sections with anti-MPO antibodies (RB-373-A, Thermo Scientific, 
Fremont, CA, USA) (34). The distribution of MPO reaction was evaluated in the liver sections. MPO-stained leukocytes were counted in 10 randomly selected areas of liver section taken from each animal of the experimental groups under a light microscope at X40 magnification. Photographs were taken with Image ProPlus (Kameram 390CU, Istanbul, Turkey).

\section{Statistical Analysis}

Significant differences within groups were estimated using oneway ANOVA with Tukey's multiple comparison test, and differences between groups were estimated using two-way ANOVA with Bonferonni posttest. $p<0.05$ was considered significant. Statistical analyses were performed with GraphPad Prism version 5.0 for Windows (GraphPad Software, San Diego, CA, USA).

\section{RESULTS}

\section{Blood Glucose Results}

Blood glucose levels are presented on Table 1. Blood glucose levels in LPS injected animals were decreased at the end of experiment (T2) compared with the control group $(p<0.001)$. Treatment of tempol did not change the glucose level compared with the LPS group. Blood glucose level in LPS + Tempol group was still lower than the control group $(p<0.001)$.

Blood glucose levels decreased during the experiment (T1 and T2) compared with the beginning of experiment (T0) in LPS $(p<0.05, p<0.001)$ and LPS + Tempol groups $(p<0.05, p<0.001)$. Blood glucose levels remained stable during the experiment in the control and tempol groups and also were similar at all three time points.

\section{Body Temperature Results}

The body temperature values are presented on Table 2. Body temperatures in LPS injected animals decreased at T2 compared with the control group $(p<0.01)$. Treatment of tempol did not prevent the reduction of body temperature and it was still lower than the control group $(p<0.001)$.

The body temperature decreased during experiment (T1 and T2) compared with the beginning of experiment (T0) in LPS $(p<0.01, p<0.01)$ and LPS + Tempol groups $(p<0.05, p<0.001)$. Body temperatures in the control and tempol groups were similar at three time points.

\section{Biochemical Results}

LPS administration increased both AST $(p<0.001)$ and ALT levels $(p<0.001)$ in the plasma and tissue. Tempol reduced AST $(p<0.001$; $p<0.05)$ and ALT $(p<0.001 ; p<0.05)$ levels in plasma and tissue of LPS injected rats. In the tempol group, while AST in plasma was similar to the control group, AST in the tissue was lower than the control group $(p<0.001)$. In this group, ALT in tissue was similar to the control group, however, ALT in the plasma was higher than in the control group $(p<0.001)$ (Figures 1,2$)$.

LPS also caused a decrease in the plasma and tissue levels of SOD compared with the control group $(p<0.001 ; p<0.05)$. Tempol administration increased SOD levels in the plasma and tissue of LPS injected animals $(p<0.001)$. In the tempol group, SOD levels in the plasma was similar to the control group, with increasing levels of SOD in liver tissue $(p<0.001)$ (Figure 3 ).

CRP, which is synthesized in liver in response to a wide variety of inflammatory stimuli, increased in the liver tissue of LPS

Table 1. Blood glucose levels (mg/dl) in the experimental groups at the three time points: T0 (the beginning of experiment), T1 (the middle of experiment) and T2 (the end of experiment). ${ }^{*} p<0.05,{ }^{* *} p<0.001$ vs. Control group. \# $p<0.05, \# \# \#<0.001$ vs. T0 baseline level.

\begin{tabular}{lccc}
\hline & T0 & T1 & T2 \\
\hline Control group & $104.50 \pm 2.69$ & $101.00 \pm 4.35$ & $97.50 \pm 3.88$ \\
\hline LPS group & $126.57 \pm 3.44^{*}$ & $95.13 \pm 9.26 \#$ & $66.38 \pm 9.26^{* * * \# \# \#}$ \\
\hline LPS + Tempol group & $123.67 \pm 3.86$ & $100.43 \pm 5.07 \#$ & $60.86 \pm 7.68^{* * * \# \# \#}$ \\
\hline Tempol group & $122.33 \pm 3.77$ & $109.714 \pm 4.89$ & $114.86 \pm 3.91$ \\
\hline
\end{tabular}

Table 2. The body temperature values in experimental groups at three time points: T0 (the beginning of experiment), T1 (the middle of experiment) and T2 (the end of experiment). ${ }^{* *} p<0.01,{ }^{* * *} p<0.001$ vs. Control group. $\# p<0.05, \# \# p<0.01, \# \# \#<<0.001$ vs. T0 baseline level.

TO

\begin{tabular}{l} 
Control group \\
LPS group \\
\hline LPS + Tempol group
\end{tabular}

T1

$37.57 \pm 0.08$

$36.94 \pm 0.14 \# \#$

$37.30 \pm 0.18 \#$

$37.47 \pm 0.11$
T2 $37.83 \pm 0.24$

$36.67 \pm 0.48^{* * \# \#}$

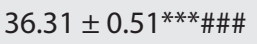



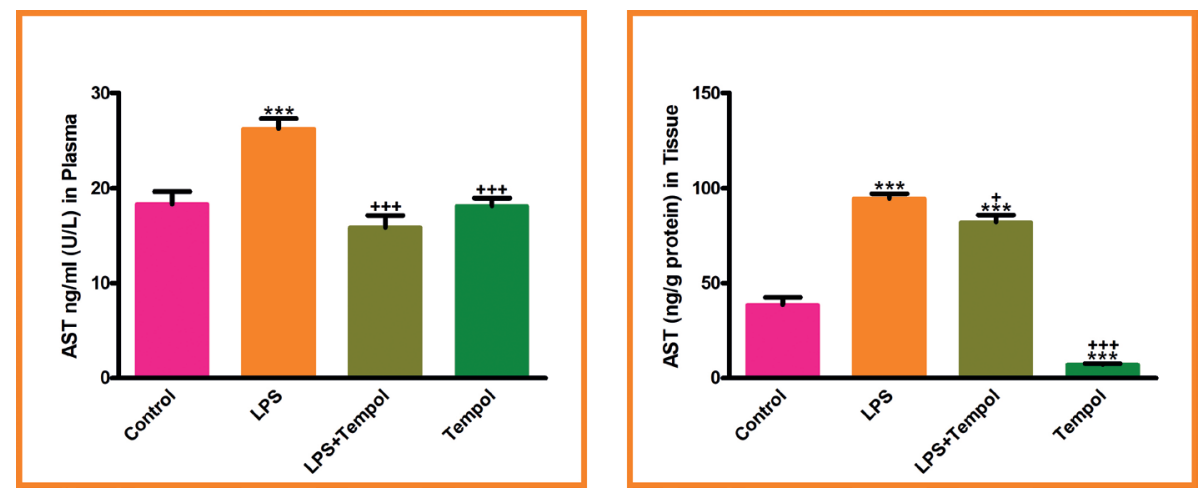

Figure 1. Aspartate aminotransferase (AST) levels in plasma and tissue of Control, LPS, LPS + Tempol and Tempol groups. ${ }^{* * *} \mathrm{p}<0.001$ vs. Control group. ${ }^{+} p<0.05,{ }^{++} p<0.001$ vs. LPS group.
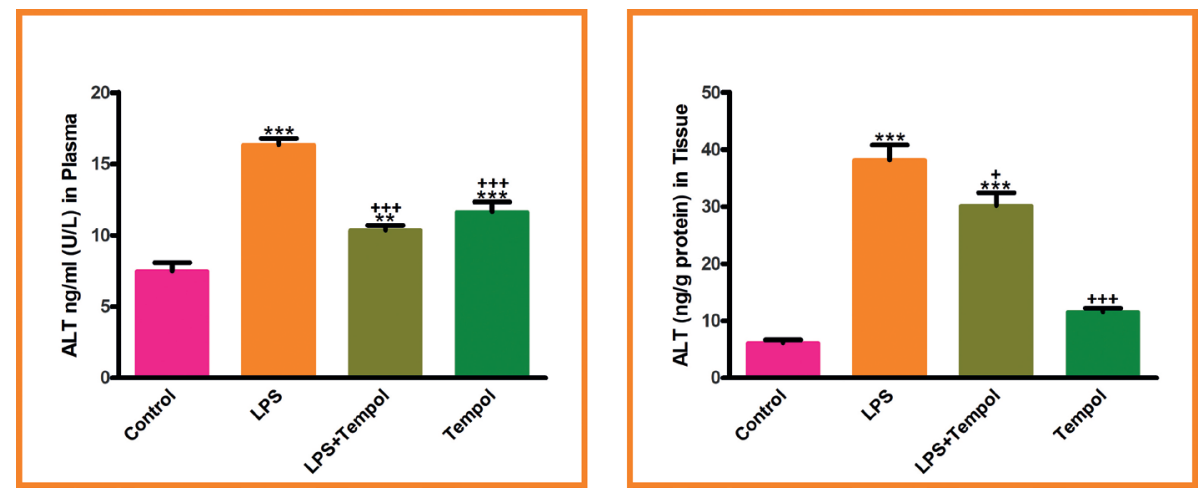

Figure 2. Alanine aminotransferase (ALT) levels in plasma and tissue of Control, LPS, LPS + Tempol and Tempol groups. ${ }^{* * *} \mathrm{p}<0.01{ }^{, * * *} \mathrm{p}<0.001$ vs. Control group. ${ }^{+} p<0.05,{ }^{+++} p<0.001$ vs. LPS group.


Figure 3. Superoxide dismutase (SOD) levels in plasma and tissue of Control, LPS, LPS + Tempol and Tempol groups. ${ }^{*} p<0.05,{ }^{, * * *} p<0.001$ vs. Control group. ${ }^{+++} \mathrm{p}<0.001$ vs. LPS group.

administrated animals $(\mathrm{p}<0.001)$. Tempol did not prevent the increase of CRP levels in LPS injected rats. In the Tempol group, CRP was similar to the control group (Figure 4).

\section{Histological Results}

Histopathological changes were shown in HE-stained sections of LPS injected animals. Dilatation of sinusoids, leukocyte infiltration, loss of outlines of hepatic plates and damage to the endothelium of central veins were seen in the LPS group. LPS leads to severe inflammatory reaction in liver parenchyma. Infiltration of many inflammatory cells was observed in liver parenchyma especially in sinusoids and vessel lumens. These inflammatory cells caused sinusoidal occlusions. In addition to these changes, structural changes in portal areas were seen in this group. Many of these results were determined in LPS + Tempol group. Tempol did not prevent inflammation and sinusoidal dilatation in the liver tissue of LPS treated animals. The histological structure of liver tissue in the group which was only given tempol was similar to the control group (Figure 5). 


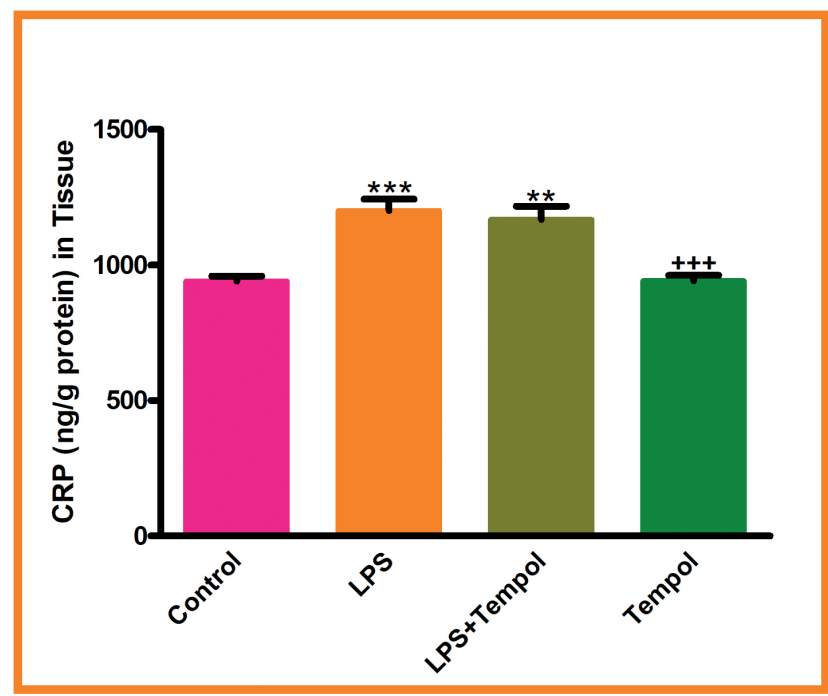

Figure 4. C-reactive protein (CRP) levels in tissue of Control, LPS, LPS + Tempol and Tempol groups. ${ }^{* *} p<0.01,{ }^{* * *} \mathrm{p}<0.001$ vs. Control group. ${ }^{++} p<0.001$ vs. LPS group.



Figure 6. MPO-stained leukocytes distributions in liver tissue of Control, LPS, LPS + Tempol and Tempol groups. ${ }^{* * *} \mathrm{p}<0.001$ vs. Control group. ${ }^{+++} p<0.001$ vs. LPS group.

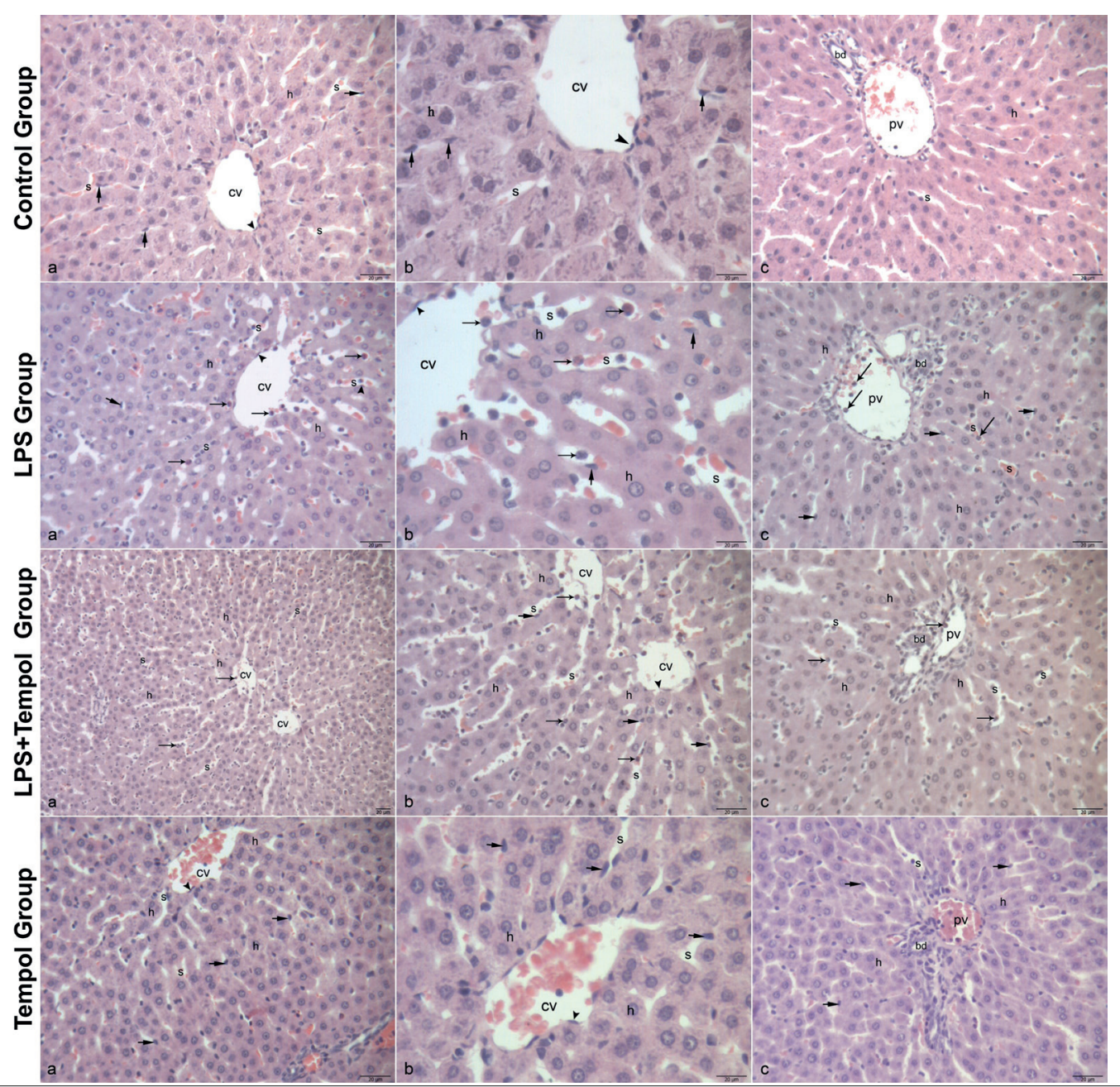

Figure 5. Central vein (cv), sinusoid (s), endothelium $(>)$, hepatocyte (h), Kupffer cell $(\rightarrow)$, leukocyte $(\rightarrow)$, portal vein $(p v)$ and bile duct (bd) on liver tissue of Control, LPS, LPS + Tempol and Tempol groups. a-c) Bar: $20 \mu \mathrm{m}, \mathrm{HE}$. 


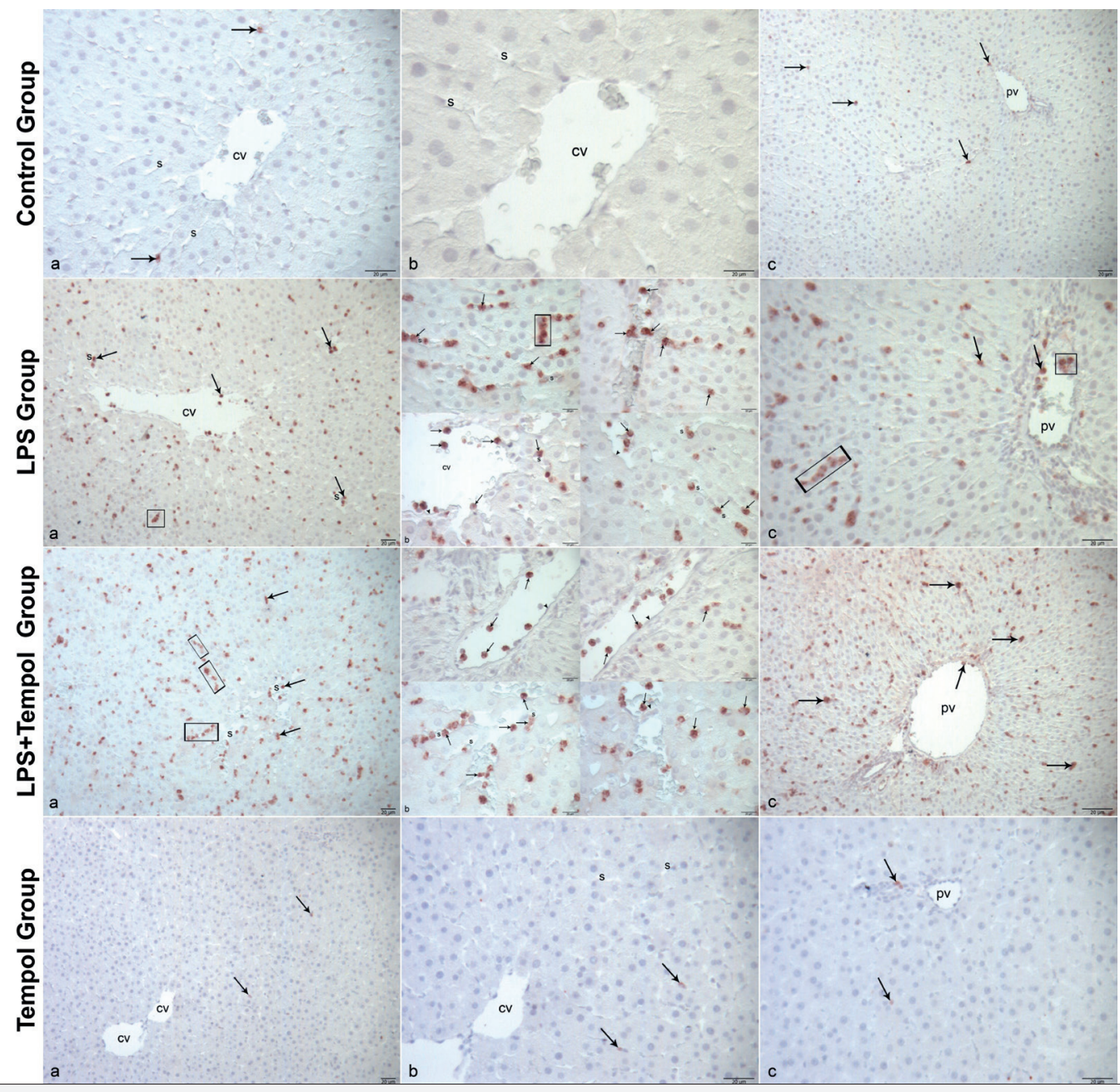

Figure 7. MPO-stained leukocytes $(\rightarrow, \square)$ in liver tissue of Control, LPS, LPS + Tempol and Tempol groups. Central vein (cv), sinusoid (s), endothelium (>) and portal vein (pv). a-c) Bar: $20 \mu \mathrm{m}$.

\section{Immunohistochemical Results}

MPO-stained leukocytes in liver tissue increased in the LPS group $(p<0.001)$ in comparison with the control. However, tempol administration did not change the distribution of MPO-stained leukocytes in LPS injected animals. MPO-stained leukocytes were seen in sinusoids, central veins and portal areas of the liver parenchyma. Infiltration of neutrophils in sinusoids led to micro abscesses formation MPO staining in tempol group was similar to control group (Figures 6, 7).

\section{DISCUSSION}

The pathophysiology of liver dysfunction in sepsis is not clearly understood (5). Liver injury in sepsis occurs not only from hypoperfusion, but also from the spread of bacteria or endotoxins, release of inflammatory cytokines and other mediators during infection (6, 9-14). Liver injury in sepsis or shock has been studied in many animal models (35). Many studies have reported that sepsis leads to liver damage, change in glucose metabolism, and increase in aminotransferase enzymes and CRP levels $(6,31,36-48)$
Cecum ligation and puncture (CLP)-induced sepsis leads to pathological changes such as necrosis, inflammation, portal inflammation in the liver of rats (36). In LPS/D-galactosamineinduced liver injury model, the liver was observed hemorrhagic necrosis and severe hepatocyte damage (37). LPS administration resulted in pathological abnormalities including hepatic edema, inflammatory cell aggregation in sinusoids and central veins at 6 hours (38). LPS-induced histopathological changes including necrosis, inflammatory cell infiltration and vacuolar degeneration were observed in the liver 4 hours after LPS injection (39). In our study, LPS administration resulted in histological changes such as hepatocytes and endothelium damage, dilatation of sinusoids, leukocyte infiltration and inflammation. The liver damage can directly contribute to liver dysfunction. It is known that AST and ALT levels in plasma and tissue are the most important markers in assessing of liver functions during experimental and clinical liver injury $(31,40)$. Many studies have reported that LPS resulted in significant elevation of ALT and AST levels (31, 38-41). In this study, LPS administration resulted in significant elevation of ALT and 
AST in plasma and liver. These results show that LPS induced liver injury and dysfunction in the experimental sepsis model.

Endotoxin causes enhanced peripheral glucose utilization, depletion of hepatic glycogen and an increase in gluconeogenesis (42). Bacterial infections influence glucose metabolism. Endotoxin results in hyperglycemia, followed by hypoglycemia (43-45). LPS causes blood glucose levels to decrease to hypoglycemic level $(43,46)$. The blood glucose level and liver glycogen decreased at $3 \mathrm{~h}$ after LPS injection. The glucose levels increased at $3 \mathrm{~h}$ after cecal incision, however, they decreased at $6 \mathrm{~h}$ after cecal incision (47). CLP caused a time-dependent increase in rectal temperature which reached the maximal at $15 \mathrm{~h}$ after CLP and then declined, whereas it caused a biphasic change in blood glucose such as hyperglycemia in the early stage (3h) and hypoglycemia in the late stage (15-18h) (27). In the present study, it was observed that LPS resulted in a decrease in the blood glucose levels at $3 \mathrm{~h}$ and $6 \mathrm{~h}$ after LPS injection. Also, body temperature decreased the same time point in LPS treated animals.

CRP is an acute phase protein synthesized from the liver. Bacterial infections result in increases in CRP levels in a few hours. CRP levels are used in the diagnosis and prognosis of infection and also sepsis $(6,48)$. In this study, LPS caused an increase in the CRP level in the liver. The increase in CRP and leukocytes infiltration indicated the inflammatory response and oxidative stress in LPS-induced experimental sepsis model. Furthermore, LPS administration decreased SOD levels in the liver and plasma.

Our findings including liver damage, leukocytes infiltration, the elevation of aminotransferases enzymes and CRP, and the decrease of SOD level indicated LPS-induced liver injury and oxidative stress in the experimental sepsis models.

The role of oxidative stress in the pathophysiology of many diseases leads to a focus on drugs that prevent the formation of ROS and to the development of treatment strategies including antioxidant enzymes such as SOD, catalase and radical scavengers. It is known that tempol prevents the formation of hydroxyl radicals by sweeping intracellular superoxide radicals and other radicals (22). Tempol is a cell membrane permeable, a radical scavenger and a SOD mimetic agent $(22,23)$. Because of these abilities and properties, tempol has been studied in animal models associated with oxidative stress (23-32). The effects of tempol on hypertension were investigated in the experimental model $(23,24)$. Schnackenberg et al., (1998) suggested that short- and long-term administration of the stable, membrane-permeable SOD mimetic tempol significantly reduces mean arterial pressure in spontaneously hypertensive rats (24). It has been shown that tempol treatment ameliorates oxidative damage in liver tissue in methotrexate-induced liver injury. Therefore, it has been suggested that tempol may be useful in protecting the liver from injury in these experimental models (25). Many previous studies have shown the beneficial effects of tempol in endotoxemia and sepsis (27-31). Liaw et al., (2005) reported that tempol $(30 \mathrm{mg} /$ kg injected 90 min after CLP and then continuously infused for $18 \mathrm{~h}$ ) not only ameliorated the deterioration of hemodynamic changes, renal and liver injuries but also attenuated neutrophil infiltration in the lung in the sepsis induced by CLP. In addition, tempol improved the survival in CLP-induced septic rats (27). It has been demonstrated that LPS caused hypotension, hepatocellular and pancreatic injury and renal dysfunction at the sixth hour of injection, and pre-treatment of tempol (100 mg/kg infusion, 15 min prior to $30 \mathrm{mg} / \mathrm{kg}$ LPS infusion) could not affect the circulatory failure, but reduced liver injury caused by LPS (31).

In our study, tempol administration reduced the AST and ALT levels in the LPS induced sepsis rats, although, these values were still higher in the tissue. These results indicated that tempol has a role in LPS-induced liver injury, but could not prevent oxidative stress and its effects. While tempol could not attenuate histological damage and leukocytes infiltration, and also did not cause any change in CRP levels, blood glucose levels and body temperature, tempol significantly increased SOD levels both in LPS-induced sepsis and in the control animals. In addition, tempol did not cause histological damage in liver tissue of the control animals. Therefore, it could be suggested that tempol may have an antioxidant effect as SOD mimetic agent. It was thought that tempol would be more effective to apply before LPS or extended to the administration time of tempol administrated 3 hours after LPS.

\section{CONCLUSION}

Results of the present study indicated that LPS induced liver injury is associated with oxidative stress and inflammation and that tempol has an antioxidant role. However, the effects of tempol were limited in this study. Therefore, further experimental studies are needed to determine the role of tempol in sepsis and shock models.

Peer-review: Externally peer-reviewed.

Author Contributions: Conception/Design of study: P.S.S., A.K., C.D.T.; Data Acquisition: P.S.S., A.K., C.D.T., T.S., H.B.; Data Analysis/ Interpretation: P.S.S., A.K., C.D.T., T.K., H.B.; Drafting Manuscript: A.K., C.D.T., E.C.B.; Critical Revision of Manuscript: A.K., C.D.T., E.C.B.; Final Approval and Accountability: P.S.S., A.K., C.D.T., E.C.B., H.B.; Technical or Material Support: P.S.S., A.K., C.D.T., T.K., H.B.; Supervision: A.K., C.D.T.

Conflict of Interest: The authors declare that they have no conflicts of interest.

Financial Disclosure: This study was funded by Scientific Research Projects Coordination Unit of Istanbul University (Project no: 27021 and 33876).

\section{REFERENCES}

1. Levy MM, Fink MP, Marshall JC, Abraham E, Angus D, Cook D, et al. 2001 SCCM/ESICM/ACCP/ATS/SIS International Sepsis Definitions Conference. Intensive Care Med 2003; 29(4): 530-8.

2. Annane D, Bellissant E, Cavaillon JM. Septic shock. Lancet 2005; 365(9453): 63-78.

3. Minasyan H. Sepsis and septic shock: Pathogenesis and treatment perspectives. J Crit Care 2017; 40: 229-42. 
4. Angus DC, van der Poll T. Severe sepsis and septic shock. N Engl J Med 2013; 369(9): 840-51.

5. Nesseler N, Launey $Y$, Aninat C, Morel F, Mallédant $Y$, Seguin P. Clinical review: The liver in sepsis. Crit Care 2012; 16(5):235.

6. Woźnica EA, Inglot M, Woźnica RK, Łysenko L. Liver dysfunction in sepsis. Adv Clin Exp Med 2018; 27(4): 547-51.

7. Koch A, Horn A, Dückers H, Yagmur E, Sanson E, Bruensing J, et al. Increased liver stiffness denotes hepatic dysfunction and mortality risk in critically ill non-cirrhotic patients at a medical ICU. Crit Care 2011; 15(6): 266.

8. Recknagel P, Gonnert FA, Westermann M, Lambeck S, Lupp A, Rudiger $A$, et al. Liver dysfunction and phosphatidylinositol-3kinase signalling in early sepsis: experimental studies in rodent models of peritonitis. PLoS Med 2012; 9(11): e1001338.

9. Wang P, Ayala A, Ba ZF, Zhou M, Perrin MM, Chaudry IH. Tumor necrosis factor-alpha produces hepatocellular dysfunction despite normal cardiac output and hepatic microcirculation. Am J Physiol1993; 265(1 Pt 1): G126-32.

10. Dhainaut JF, Marin N, Mignon A, Vinsonneau C. Hepatic response to sepsis: interaction between coagulation and inflammatory processes. Crit Care Med 2001; 29(7 Suppl): 42-7.

11. Garofalo AM, Lorente-Ros M, Goncalvez G, Carriedo D, BallénBarragán A, Villar-Fernández A, et al. Histopathological changes of organ dysfunction in sepsis. Intensive Care Med Exp 2019; 7(Suppl 1):45.

12. Mannaa, FA, Abdel-Wahhab, KG. Physiological potential of cytokines and liver damages. Hepatoma Res 2016; 2: 131-43.

13. Szabo G, Romics L Jr, Frendl G. Liver in sepsis and systemic inflammatory response syndrome. Clin Liver Dis 2002; 6(4): 104566.

14. Strnad P, Tacke F, Koch A, Trautwein C. Liver - guardian, modifier and target of sepsis. Nat Rev Gastroenterol Hepatol 2017; 14(1): 55-66.

15. Ring A, Stremmel W. The hepatic microvascular responses to sepsis. Semin Thromb Hemost 2000; 26(5): 589-94.

16. Wong CH, Jenne CN, Petri B, Chrobok NL, Kubes P. Nucleation of platelets with blood-borne pathogens on Kupffer cells precedes other innate immunity and contributes to bacterial clearance. Nat Immunol 2013; 14(8): 785-92.

17. Komine S, Akiyama K, Warabi E, Oh S, Kuga K, Ishige K, et al. Exercise training enhances in vivo clearance of endotoxin and attenuates inflammatory responses by potentiating Kupffer cell phagocytosis. Sci Rep 2017; 7(1): 11977.

18. Koo DJ, Chaudry IH, Wang P. Kupffer cells are responsible for producing inflammatory cytokines and hepatocellular dysfunction during early sepsis. J Surg Res 1999; 83(2): 151-7.

19. McCuskey RS, Nishida J, McDonnell D. Effect of immunoglobulin $G$ on the hepatic microvascular inflammatory response during sepsis. Shock 1996; 5 (1): 28-33.

20. Fridovich I. Superoxide radical and superoxide dismutases. Annu Rev Biochem 1995; 64: 97-112.

21. Fridovich I. Superoxide dismutases. An adaptation to a paramagnetic gas. J Biol Chem 1989; 264(14): 7761-4.

22. Samuni A, Krishna CM, Riesz P, Finkelstein E, Russo A. A novel metal-free low molecular weight superoxide dismutase mimic. J Biol Chem 1988; 263(34): 17921-4.

23. Wilcox CS. Effects of tempol and redox-cycling nitroxides in models of oxidative stress. Pharmacol Ther 2010; 126(2): 119-45.

24. Schnackenberg CG, Welch WJ, Wilcox CS. Normalization of blood pressure and renal vascular resistance in SHR with a membranepermeable superoxide dismutase mimetic: role of nitric oxide. Hypertension 1998; 32(1): 59-64.
25. Pınar N, Kaplan M, Özgür T, Özcan O. Ameliorating effects of tempol on methotrexate-induced liver injury in rats. Biomed Pharmacother 2018; 102: 758-64.

26. Ergin B, Bezemer R, Kandil A, Demirci-Tansel C, Ince C. TEMPOL has limited protective effects on renal oxygenation and hemodynamics but reduces kidney damage and inflammation in a rat model of renal ischemia/reperfusion by aortic clamping. J Clin Transl Res 2015; 1(2): 1-13.

27. Liaw WJ, Chen TH, Lai ZZ, Chen SJ, Chen A, Tzao C, et al. Effects of a membrane-permeable radical scavenger, Tempol, on intraperitoneal sepsis-induced organ injury in rats. Shock 2005; 23(1): 88-96.

28. Wang W, Zolty E, Falk S, Summer S, Zhou Z, Gengaro P, et al. Endotoxemia-related acute kidney injury in transgenic mice with endothelial overexpression of GTP cyclohydrolase-1. Am J Physiol Renal Physiol 2008; 294(3): F571-6.

29. Wang W, Jittikanont S, Falk SA, Li P, Feng L, Gengaro PE, et al. Interaction among nitric oxide, reactive oxygen species, and antioxidants during endotoxemia-related acute renal failure. Am J Physiol Renal Physiol 2003; 284(3): F532-7.

30. Zacharowski K, Olbrich A, Cuzzocrea S, Foster SJ, Thiemermann C. Membrane-permeable radical scavenger, tempol, reduces multiple organ injury in a rodent model of gram-positive shock. Crit Care Med 2000; 28(6): 1953-61.

31. Leach M, Frank S, Olbrich A, Pfeilschifter J, Thiemermann C. Decline in the expression of copper/zinc superoxide dismutase in the kidney of rats with endotoxic shock: effects of the superoxide anion radical scavenger, tempol, on organ injury. $\mathrm{Br} J$ Pharmacol 1998; 125(4): 817-25.

32. Yuksel BC, Serdar SE, Tuncel A, Uzum N, Ataoglu O, Atan A, et al. Effect of tempol, a membrane-permeable radical scavenger, on mesenteric blood flow and organ injury in a murine cecal ligation and puncture model of septic shock. Eur Surg Res 2009; 43(2): 21927.

33. Demirci C, Gargili A, Kandil A, Cetinkaya H, Uyaner I, Boynuegri B, et al. Inhibition of inducible nitric oxide synthase in murine visceral larva migrans: effects on lung and liver damage. Chin J Physiol 2006; 49(6): 326-34.

34. Legrand M, Almac E, Mik EG, Johannes T, Kandil A, Bezemer R, et al. L-NIL prevents renal microvascular hypoxia and increase of renal oxygen consumption after ischemia-reperfusion in rats. Am J Physiol Renal Physiol 2009; 296(5): F1109-17.

35. Lilley E, Armstrong R, Clark N, Gray P, Hawkins P, Mason K, et al. Refinement of animal models of sepsis and septic shock. Shock 2015; 43(4): 304-16.

36. Muftuoglu MA, Aktekin A, Ozdemir NC, Saglam A. Liver injury in sepsis and abdominal compartment syndrome in rats. Surg Today 2006; 36(6): 519-24.

37. Li G, Liu Y, Tzeng NS, Cui G, Block ML, Wilson B, et al. Protective effect of dextromethorphan against endotoxic shock in mice. Biochem Pharmacol 2005; 69(2): 233-40.

38. Liu X, Liu R, Dai Z, Wu H, Lin M, Tian F, et al. Effect of Shenfu injection on lipopolysaccharide (LPS)-induced septic shock in rabbits. J Ethnopharmacol 2019; 234: 36-43.

39. Sha J,Zhang H, ZhaoY, Feng X, HuX, Wang C, etal. Dexmedetomidine attenuates lipopolysaccharide-induced liver oxidative stress and cell apoptosis in rats by increasing GSK-3 $3 /$ MKP-1/Nrf2 pathway activity via the a2 adrenergic receptor. Toxicol Appl Pharmacol 2019; 364: 144-52.

40. Zhou R, Chen SH, Li G, Chen HL, Liu Y, Wu HM, et al. Ultralow doses of dextromethorphan protect mice from endotoxin-induced sepsis-like hepatotoxicity. Chem Biol Interact 2019; 303: 50-6. 
41. Thiemermann C, Ruetten $\mathrm{H}, \mathrm{Wu}$ CC, Vane JR. The multiple organ dysfunction syndrome caused by endotoxin in the rat: attenuation of liver dysfunction by inhibitors of nitric oxide synthase. $\mathrm{Br} J$ Pharmacol 1995; 116(7): 2845-51.

42. Mészáros K, Lang CH, Bagby GJ, Spitzer JJ. Contribution of different organs to increased glucose consumption after endotoxin administration. J Biol Chem 1987; 262(23): 10965-70.

43. Engin A, Zemheri M, Bukan N, Memiş L. Effect of nitric oxide on the hypoglycaemic phase of endotoxaemia. ANZ J Surg 2006; 76(6): 512-7.

44. Wallington J, Ning J, Titheradge MA. The control of hepatic glycogen metabolism in an in vitro model of sepsis. Mol Cell Biochem 2008; 308(1-2): 183-92.

45. Yelich MR, Witek-Janusek L. Glucose, lactate, insulin and somatostatin responses to endotoxin in developing rats. Shock 1994; 2: 438-44.
46. Anavi S, Hahn-Obercyger M, Margalit R, Madar Z, Tirosh O. A novel antihypoglycemic role of inducible nitric oxide synthase in liver inflammatory response induced by dietary cholesterol and endotoxemia. Antioxid Redox Signal 2013; 19(16): 1889-901.

47. Chang CK, Gatan M, Schumer W. Efficacy of anti-tumor necrosis factor polyclonal antibody on phosphoenolpyruvate carboxykinase expression in septic and endotoxemic rats. Shock 1996; 6(1): 57-60.

48. Rello J, Valenzuela-Sánchez F, Ruiz-Rodriguez M, Moyano S. Sepsis: A review of advances in management. Adv Ther 2017; 34(11): 2393-411. 
